\title{
Quantum theory of the dispersion of the laser light
}

\author{
Miroslav Pardy \\ Department of Physical Electronics \\ Faculty of Science, Masaryk University \\ Kotlářská 2, 61137 Brno, Czech Republic \\ e-mail:pamir@physics.muni.cz
}

November 21, 2018

\begin{abstract}
We derive the index of refraction of light from quantum theory of atoms and from the Dirac equation with the plane wave. The result is the integral a part of the mainstream of the quantum optics. The article involves also discussion on the possibility to create the electron-positron pairs during the Čerenkov process with the adequate intex of refraction.
\end{abstract}

\section{Introduction}

The refractive index of materials varies with the frequency of light. This is called dispersion and causes prisms and rainbows to divide white light into its constituent spectral colors. This effect was described by Jan Marcus Marci (1668) and Newton (1686) in the old age of optics.

The contemporary explanation of this effect is as follows. An electromagnetic wave phase velocity is slowed in a medium because the electric field creates a disturbance in the charges of each atom (primarily the electrons). As the electromagnetic fields oscillate in the wave, the charges in the material will be oscillating at the same frequency.

The charges thus radiate their own electromagnetic wave at the same frequency, but usually with a phase delay, as the charges may move out of phase with the force driving them. The light wave traveling in the medium is the macroscopic superposition of the original wave plus the waves radiated by all the moving charges. This wave is typically a wave with the same frequency but shorter wavelength than the original, leading to a slowing of the wave phase velocity. Most of the radiation from oscillating material charges will modify the incoming wave, changing its velocity. 


\section{The quantum theory dispersion}

We suppose that electrons in atoms are in the same quantum state. The perturbation method is adequate for the application in a medium because the interaction energy of atoms with the external field is very small in comparison with the energy of electrons in atoms.

The impinging wave of electromagnetic field is of the form $E=E_{0} \cos (\omega t-2 \pi x / \lambda)$. With regard to the fact that $\lambda \sim 10^{-5} \mathrm{~cm}$ and atom is of the size $a \sim 10^{-8} \mathrm{~cm}$, the quantity $x / \lambda$ can be neglected in the electromagnetic wave and we write

$$
E=E_{0} \cos (\omega t-2 \pi x / \lambda) \rightarrow E=E_{0} \cos (\omega t)
$$

So, the quantum theory of dispersion can be derived in the framework of the nonrelativistic Schrödinger equation (Sokolov et al., 1962) for an electron moving in dielectric medium and in the field with the periodic force

$$
F_{x}=-e E_{0} \cos \omega t, \quad F_{y}=F_{z}=0 .
$$

Then, the corresponding potential energy is

$$
V^{\prime}=e x E_{0} \cos \omega t
$$

and this potential energy is the perturbation energy in the Schrödinger equation

$$
\left(i \hbar \frac{\partial}{\partial t}-H_{0}-V^{\prime}\right) \psi_{k}(t)=0
$$

where for $V^{\prime}=0$ it is $\psi_{k}(t) \rightarrow \psi_{k}^{0}(t)$ and

$$
\psi_{k}^{0}(t)=\psi_{k}^{0} e^{-\frac{i}{\hbar} E_{k} t}=\psi_{k}^{0} e^{-i \omega_{k} t},
$$

where $\psi_{k}^{0}$ is the solution of the Schrödinger equation without perturbation, or,

$$
\left(i \hbar \frac{\partial}{\partial t}-H_{0}\right) \psi_{k}^{0}(t)=0
$$

We are looking for the solution of the Schrödinger equation involving the perturbation potential in the form

$$
\psi_{k}(t)=\psi_{k}^{0}(t)+\psi_{k}^{1}(t)
$$

where $\psi_{k}^{1}(t)$, is the perturbation wave function correction to the non-perturbation wave function.

After insertion of formula (7) to eq. (4), we get

$$
\left(i \hbar \frac{\partial}{\partial t}-H_{0}\right) \psi_{k}^{1}(t)=\frac{1}{2} e x E_{0} \psi_{k}^{0}\left(e^{-i t\left(\omega_{k}-\omega\right)}+e^{-i t\left(\omega_{k}+\omega\right)}\right) .
$$

Let us look for the solution of eq. (8) in the form: 


$$
\psi_{k}^{1}(t)=u e^{-i t\left(\omega_{k}-\omega\right)}+v e^{-i t\left(\omega_{k}+\omega\right)} .
$$

After insertion of (9) into (8), we get two equations for $u$ and $v$ :

$$
\begin{aligned}
& \left(\hbar\left(\omega_{k}-\omega\right)-H_{0}\right) u=\frac{1}{2} e x E_{0} \psi_{k}^{0}, \\
& \left(\hbar\left(\omega_{k}+\omega\right)-H_{0}\right) v=\frac{1}{2} e x E_{0} \psi_{k}^{0} .
\end{aligned}
$$

Then, using the formal expansion

$$
u=\sum_{k^{\prime \prime}} C_{k^{\prime \prime}} \psi_{k^{\prime \prime}}^{0}
$$

we get from eq.

$$
\left(E_{k^{\prime \prime}}-H_{0}\right) \psi_{k^{\prime \prime}}^{0}=0
$$

the following equation

$$
\hbar \sum_{k^{\prime \prime}} C_{k^{\prime \prime}}\left(\omega_{k k^{\prime \prime}}-\omega\right) \psi_{k^{\prime \prime}}^{0}=\frac{e x E_{0}}{2} \psi_{k}^{0}
$$

with

$$
\omega_{k k^{\prime \prime}}=\frac{E_{k}-E_{k^{\prime \prime}}}{\hbar}
$$

Using the orthogonal relation

$$
\int \psi_{k^{\prime}}^{0 *} \psi_{k^{\prime \prime}}^{0} d^{3} x=\delta_{k^{\prime} k^{\prime \prime}}
$$

we get the following relation for $C_{k}$ and $u$ as follows:

$$
\begin{gathered}
C_{k}=-\frac{e E_{0}}{2 \hbar} \cdot \frac{x_{k^{\prime} k}}{\omega_{k^{\prime} k}+\omega}, \\
u=\sum_{k^{\prime}}\left(-\frac{e E_{0}}{2 \hbar}\right) \cdot \frac{x_{k^{\prime} k}}{\omega_{k^{\prime} k}+\omega} \psi_{k^{\prime}}^{0}
\end{gathered}
$$

and $v=u(-\omega)$, or

$$
v=\sum_{k^{\prime}}\left(-\frac{e E_{0}}{2 \hbar}\right) \cdot \frac{x_{k^{\prime} k}}{\omega_{k^{\prime} k}-\omega} \psi_{k^{\prime}}^{0}
$$

and

$$
x_{k^{\prime} k}=\int \psi_{k^{\prime}}^{0 *} x \psi_{k}^{0} d^{3} x .
$$

The general wave function can be obtained from eqs. (7), (9), (18) and (19) in the form: 


$$
\begin{gathered}
\psi_{k}(t)=e^{-i \omega_{k} t} \times \\
\left\{\psi_{k}^{0}-\frac{e E_{0}}{\hbar} \sum_{k^{\prime}} \frac{x_{k^{\prime} k}}{\omega_{k^{\prime} k}^{2}-\omega^{2}} \psi_{k^{\prime}}^{0}\left[\omega_{k^{\prime} k} \cos \omega t-i \omega \sin \omega t\right]\right\} .
\end{gathered}
$$

The classical polarization of a medium is given by the well known formula

$$
P=N p=-N e x,
$$

where $\mathrm{N}$ is the number of atom in the unite volume of dielectric medium. So we are able to define the quantum analogue form of the polarization as it follows:

$$
P=N \bar{p}=-N e \int \psi_{k}^{*}(t) x \psi_{k}(t) d^{3} x,
$$

or, with

$$
\int \psi_{k}^{0 *} x \psi_{k}^{0} d^{3} x=0,
$$

we have

$$
P=\sum_{k^{\prime}}\left(2 \frac{N e^{2} E_{0}}{\hbar}\right) \cdot \frac{\omega_{k^{\prime} k}\left|x_{k^{\prime} k}\right|^{2}}{\omega_{k^{\prime} k}^{2}-\omega^{2}} \cos \omega t .
$$

Using the classical formula for polarization $P$,

$$
\mathbf{P}=\frac{n^{2}-1}{4 \pi} \mathbf{E},
$$

we get for the quantum model of polarization

$$
\frac{n^{2}-1}{4 \pi}=\sum_{k^{\prime}}\left(2 \frac{N e^{2}}{\hbar}\right) \cdot \frac{\omega_{k^{\prime} k}\left|x_{k^{\prime} k}\right|^{2}}{\omega_{k^{\prime} k}^{2}-\omega^{2}} .
$$

Using the definition of the coefficients $f_{k^{\prime} k}$ by relation

$$
f_{k^{\prime} k}=\frac{2 m}{\hbar} \omega_{k^{\prime} k}\left|x_{k^{\prime} k}\right|^{2},
$$

we get the modified equation (27) as follows:

$$
\frac{n^{2}-1}{4 \pi}=\frac{N e^{2}}{m} \sum_{k^{\prime}} \frac{f_{k^{\prime} k}}{\omega_{k^{\prime} k}^{2}-\omega^{2}} .
$$

The last formula should be compared with the classical one:

$$
\frac{n^{2}-1}{4 \pi}=\frac{e^{2}}{m} \sum_{k} \frac{N_{k}}{\omega_{k}^{2}-\omega^{2}},
$$

where $N_{k}$ is number of electrons moving with frequency $\omega_{k}$ in the unit volume.

Let us renark that the oscillator coeficients $f_{k^{\prime} k}$ in eq. (29) can have also the negative values. It leads to the special behaviour of the dispersion. Namely, dispersion is negative. The negative dispersion was discovered by Ladenburg $(1921 ; 1930)$. 


\section{Volkov solution of the Dirac equation}

We follow the method of derivation and metric convention of (Berestetsky et al., 1989):

$$
(\gamma(p-e A)-m) \psi=0
$$

where

$$
A^{\mu}=A^{\mu}(\varphi) ; \quad \varphi=k x=\omega t-\mathbf{k x}
$$

We suppose that the four-potential satisfies the Lorentz gauge condition

$$
\partial_{\mu} A^{\mu}=k_{\mu}\left(A^{\mu}\right)^{\prime}=\left(k_{\mu} A^{\mu}\right)^{\prime}=0,
$$

where the prime denotes derivative with regard to $\varphi$. From the last equation follows

$$
k A=\text { const }=0,
$$

because we can put the constant to zero. The tensor of electromagnetic field is

$$
F_{\mu \nu}=k_{\mu} A_{\nu}^{\prime}-k_{\nu} A_{\mu}^{\prime}
$$

Instead of the linear Dirac equation (31), we consider the quadratic equation, which we get by multiplication of the linear equation by operator $(\gamma(p-e A)+m)$ (Berestetzkii et al., 1989). We get:

$$
\left[(p-e A)^{2}-m^{2}-\frac{i}{2} e F_{\mu \nu} \sigma^{\mu \nu}\right] \psi=0 .
$$

Using $\partial_{\mu}\left(A^{\mu} \psi\right)=A^{\mu} \partial_{\mu} \psi$, which follows from eq. (34), and $\partial_{\mu} \partial^{\mu}=\partial^{2}=-p^{2}$, with $p_{\mu}=i\left(\partial / \partial x^{\mu}\right)=i \partial_{\mu}$, we get the quadratic Dirac equation for the four potential of the plane wave:

$$
\left[-\partial^{2}-2 i e(A \partial)+e^{2} A^{2}-m^{2}-i e(\gamma k)\left(\gamma A^{\prime}\right)\right] \psi=0 .
$$

We are looking for the solution of the last equation in the form:

$$
\psi=e^{-i p x} F(\varphi) .
$$

After insertion of eq. (33) into eq. (32), we get with $\left(k^{2}=0\right)$

$$
\partial^{\mu} F=k^{\mu} F^{\prime}, \quad \partial_{\mu} \partial^{\mu} F=k^{2} F^{\prime \prime}=0,
$$

the following equation for $F(\varphi)$

$$
2 i(k p) F^{\prime}+\left[-2 e(p A)+e^{2} A^{2}-i e(\gamma k)\left(\gamma A^{\prime}\right)\right] F=0 .
$$

The integral of the last equation is of the form (Berestetzkii et al., 1989): 


$$
F=\exp \left\{-i \int_{0}^{k x}\left[\frac{e(p A)}{(k p)}-\frac{e^{2}}{2(k p)} A^{2}\right] d \varphi+\frac{e(\gamma k)(\gamma A)}{2(k p)}\right\} \frac{u}{\sqrt{2 p_{0}}},
$$

where $u / \sqrt{2 p_{0}}$ is the arbitrary constant bispinor.

Al powers of $(\gamma k)(\gamma A)$ above the first are equal to zero, since

$$
\begin{gathered}
(\gamma k)(\gamma A)(\gamma k)(\gamma A)= \\
-(\gamma k)(\gamma k)(\gamma A)(\gamma A)+2(k A)(\gamma k)(\gamma A)=-k^{2} A^{2}=0 .
\end{gathered}
$$

where we have used eq. (4) and relation $k^{2}=0$. Then we can write:

$$
\exp \left\{e \frac{(\gamma k)(\gamma A)}{2(k p)}\right\}=1+\frac{e(\gamma k)(\gamma A)}{2(k p)} .
$$

So, the solution is of the form:

$$
\psi_{p}=R \frac{u}{\sqrt{2 p_{0}}} e^{i S}=\left[1+\frac{e}{2 k p}(\gamma k)(\gamma A)\right] \frac{u}{\sqrt{2 p_{0}}} e^{i S},
$$

where $u$ is an electron bispinor of the corresponding Dirac equation

$$
(\gamma p-m) u=0
$$

and we shall take it to be normalized by condition $\bar{u} u=2 \mathrm{~m}$. The mathematical object $S$ is the classical Hamilton-Jacobi function, which was determined in the form:

$$
S=-p x-\int_{0}^{k x} \frac{e}{(k p)}\left[(p A)-\frac{e}{2} A^{2}\right] d \varphi .
$$

The current density is

$$
j^{\mu}=\bar{\psi}_{p} \gamma^{\mu} \psi_{p}
$$

where $\bar{\Psi}$ is defined as the transposition of (44), or,

$$
\bar{\psi}_{p}=\frac{\bar{u}}{\sqrt{2 p_{0}}}\left[1+\frac{e}{2 k p}(\gamma A)(\gamma k)\right] e^{-i S} .
$$

After insertion of $\Psi_{p}$ and $\bar{\Psi}_{p}$ into the current density, we have:

$$
j^{\mu}=\frac{1}{p_{0}}\left\{p^{\mu}-e A^{\mu}+k^{\mu}\left(\frac{e(p A)}{(k p)}-\frac{e^{2} A^{2}}{2(k p)}\right)\right\} .
$$




\section{Index of refraction from the Volkov solution of the Dirac equation}

Let be $\psi_{a}$ the wave function of the electron in the vector field potential in an atom (a) $A_{a}$, and $\Psi_{w}$ the wave function of the electron in the plane wave (w) potential $A_{w}$. The corresponding Dirac equations of these two situations are as follows:

$$
\begin{aligned}
& \left(\gamma\left(p-e A_{a}\right)-m\right) \psi_{a}=0 . \\
& \left(\gamma\left(p-e A_{v}\right)-m\right) \psi_{w}=0 .
\end{aligned}
$$

If we take the sum of eqs. (50) and (51), we get

$$
\left(\gamma\left(p-e A_{a}\right)\right) \psi_{a}+\left(\gamma\left(p-e A_{w}\right)\right) \psi_{w}=m\left(\psi_{a}+\psi_{w}\right)
$$

which is not equation of the total system with potentials $A_{a}+A_{w}$.

It may be easy to see that the Dirac equation for the sum $\psi_{a}+\psi_{w}$ is the following equation:

$$
\left(\gamma\left(p-e\left(A_{a}+A_{w}\right)\right)\left(\psi_{a}+\psi_{w}\right)=m\left(\psi_{a}+\psi_{w}\right)-A_{a} \psi_{w}-A_{w} \psi_{a},\right.
$$

or, wave function $\left(\psi_{a}+\psi_{w}\right)$ is not solution of the Dirac equation for the complex system $\left(A_{a}+A_{w}\right)$.

Nevertheless, we can use the wave function $\psi=\psi_{a}+\psi_{v}$ as the first approximation in determinantion of the index of refraction for the system, which is in the potential $A_{a}$ and in the wave potential $A_{v}$.

With regard to eq. (26), we write for the polarization

$$
\mathbf{P}=N e \int \bar{\psi} \mathbf{x} \psi=\frac{n^{2}-1}{4 \pi} \mathbf{E}=\frac{n^{2}-1}{4 \pi}\left\{-\frac{1}{c} \frac{\partial \mathbf{A}}{\partial t}-\operatorname{grad} \varphi\right\},
$$

where we used the definition of $\mathbf{E}$ according to the Landau et al. textbook (Landau et al., 1988).

So, we get the original formula for the intex of refraction of medium in case the signal propagatiin throught the medium is the plane wave:

$$
\frac{n^{2}-1}{4 \pi}=\frac{N e \int \bar{\psi} \mathbf{x} \psi}{-\frac{1}{c} \frac{\partial \mathbf{A}}{\partial t}-\operatorname{grad} \varphi}
$$

where $\psi=\psi_{a}+\psi_{w}$.

\section{Discussion}

The last formula (55) is original and it is not excluded that it will play the crucial role in modern optics. It is possible expect the application of it in the graphene physics (Novoselov et al., 2005) where every new result in quantum optics is valuable. At the same time we hope that the derived formula will be tested by the greatist laser system over the world, called ELI. 
The article is related also to the pair production by the Cerenkov process. Pair production is the creation of a particle and its antiparticle from a neutral boson. Examples are - creating an electron and a positron, a muon and an antimuon, a proton and an antiproton and so on. In order for pair production to occur, the incoming energy of the interaction must be abovet he total rest mass energy of the two particles. Both energy and momentum are conserved. However, all other conserved quantum numbers (angular momentum, electric charge, lepton number) of the produced particles must sum to zero.

The Čerenkov radiation is emitted by motion of fast charged particle in a medium when its speed is faster than the speed of light in this medium This effect was observed experimentally first by Cerenkov $(1934 ; 1936)$ and theoretically interpreted by Frank and Tamm (1937) in the framework of the of the clasical electrodynamics. The modern field theory explanation was given by Schwinger et al. (1976) and the particle production by the Cerenkov mechanism including temperature was discussed by Pardy (1983a; 1983b; 1989; 1994; 2002) and experts in particle physics.

The spectrum of radiation is modified involving the radiation correction in the photon propagator (Pardy, 1994). The experimental value for pair creation is (Pardy, 1994) for

$$
\omega^{2}>\frac{4 m^{2} c^{2} v^{2}}{\hbar^{2}\left(n^{2} \beta^{2}-1\right)}
$$

It means that for $n=\sqrt{2}$ and $v \approx c$, we get from eq. (56) $\hbar \omega \approx 2 m c^{2}$, which can be interpreted as the condition for creation of the electron-positron pair by the gamma quantum.

The condition (56) is related to the gamma photons rather than to the optical ones. The possibility of the existence of the gamma Cerenkov radiation is discussed by Ion and Stocker (1993) in nuclear physics. The so called nuclear gamma Čerenkov radiation requires a special experimental technique in order to extract such radiation from the background produced by other mechanism. Such techniques are well known in nuclear physics and we can expect that sooner or later the existence of the gamma Cerenkov radiation in nuclear physics will be confirmed together with the pair production.

The present theory can be generalized to the the string-like objects (Manoukian, 1991; 1992) where the problems of particle production by strings are discussed.

\section{REFERENCES}

Berestetzkii, V. B.; Lifshitz, E. M. and Pitaevskii, L. P. Quantum electrodynamics, (Moscow, Nauka, 1989). (in Russian).

Črenkov. P. A. (1934). The visible radiation of pure liquids caused by $\gamma$-rays, Comptes Rendus Hebdomaclaires des Séances de l'Académie des Sciences USSR 2, 451.

Čerenkov, P. A. (1936). Visible Radiation Produced by Electrons Moving in a Medium with Velocities Exceeding that of Ligh C. R. Acad. Sci. (USSR) 3, 413. 
Ion, D. B. and Stocker, W. (1993). High-energy nuclear $\gamma$-Cherenkov radiation, Phys. Lett. B 311,339 .

Ladenburg, R. (1921). Die quantentheoretische Bedeutung der Zahl der Dispersionelektronen, Zeitschrift für Physik, 4, No. 4, 451-468 (1921).

Ladenburg, . and Levy, S. (1930). Untersuchungen ber die anomale Dispersion angeregter Gase VI. Teil: Kontrollversuche für den Nachweis der negativen Dispersion: Absorption, anomale Dispersion, Intensittsverteilung und Intensität verschiedener Neonlinien, Zeitschrift für Physik, 65, Nos. 3-4. pp. 189-206.

Landau, L. D. and Lifshitz, E. M., The classical theory of fields, 7th ed., (Moscow, Nauka, 1988), (in Russian).

Manoukian, E. B. (1991). Electromagnetic radiation from a Nambu string at finite temperatures, Nuovo Cimento 104 A, No. 9, 1409.

Manoukian, E. B. (1992). $e e^{+}$Production by a Nambu string, International Journal of Theoretical Physics 31, No. 6, 1003.

Marci, J. M. (Johannes Marcus Marci de Cronland), (1668). De arcu coelesti, (Charles University, Prague)

Newton, I. (1998). Opticks: or, a treatise of the reflexions, refractions, inflexions and colours of light. Also two treatises of the species and magnitude of curvilinear figures. Commentary by Nicholas Humez (Octavo ed.). Palo Alto, Calif.: (Opticks was originally published in 1704).

Novoselov, K.S.; Geim, A. K.; Morozov, S.V. et al. (2005). Two-dimensional gas of massless Dirac fermions in graphene, Nature, 438, 197.

Pardy, M. (1983a). Particle production by the Čerenkov mechanism, Phys. Lett. 94 A, 30.

Pardy, M. (1983b). Particle production by accelerated charges moving in condensed matter, Journal of Phys. G: Nucl. Phys. 9853.

Pardy, M. (1989). Finite temperature Čerenkov radiation, Phys. Lett. A 134, No. 6, 357.

Pardy, M. (1994). The Čerenkov effect with radiative corrections, Phys. Lett. B 325, 517.

Pardy, M. (2002). Čerenkov effect with massive photons, Int. Journal of Theoretical Physics 41 No. 5,887 .

Sokolov, A. A.; Loskutov, Yu. M. and Ternov, I.M. Quantum mechanics, (Moscow , 1962). (in Russian).

Schwinger, J., Tsai, W. Y. and Erber, T. (1976). Classical and quantum theory of synergic synchrotron-Čerenkov radiation, Annals of Physics (NY) 96, 303.

Tamm, I. E. and Frank, I. M. (1937). The coherent radiation of a fast electron in a medium, Dokl. Akad. Nauk SSSR 14, 109. 